\title{
Evaluación pretrasplante en otorrinolaringología
}

\section{Pretransplant evaluation in otolaryngology}

\author{
Carolina Oliva G. ${ }^{1}$, Paul Boettiger B. ${ }^{1,2}$, Lara Fernández R..$^{1,3}$
}

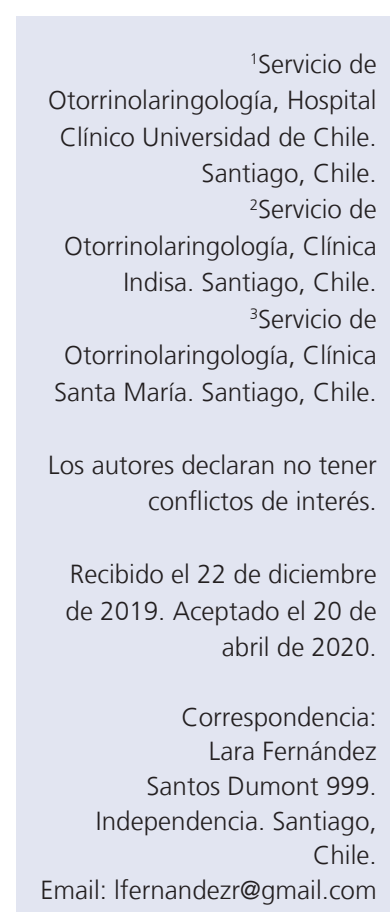

\section{Introducción}

Los pacientes trasplantados presentan mayor vulnerabilidad a complicaciones infecciosas, no solo debido al uso de drogas inmunosupresoras, sino que también, a las enfermedades subyacentes que presentan y a la falla de órganos primarios ${ }^{1}$. En estudios retrospectivos se estima que entre el 15\% al $35 \%$ de los pacientes presenta infecciones antes de recibir el trasplante, destacando neumonía, sepsis, celulitis, infecciones del tracto urinario, infecciones asociadas a catéteres vasculares, peritonitis bacteriana espontánea, entre otras.
En estos casos al recibir un tratamiento adecuado y oportuno no existe una diferencia en el resultado ni aumenta la mortalidad luego del trasplante $e^{2-4}$. Si bien, las infecciones otorrinolaringológicas no son frecuentes en estos pacientes, tanto en la etapa pre como postrasplante, es importante establecer un adecuado estudio y tratamiento de ellas debido a que las infecciones sin control pueden ser una contraindicación para el proceso de trasplante, además de posible riesgo de complicación tras inmunosupresión e infecciones silentes ${ }^{2}$.

El objetivo de este artículo es aportar directrices en el estudio pretrasplante desde 
un enfoque otorrinolaringológico, generando recomendaciones de acuerdo a la patología del paciente y el órgano a trasplantar. Se utilizará la evidencia disponible hasta el momento con el fin de disminuir la cantidad de procedimientos y exámenes innecesarios, minimizando los tiempos y costos asociados, dando una guía y flujograma para que el enfrentamiento de estos pacientes sea óptimo.

\section{Evaluación rinosinusal pretrasplante}

Dividiremos patología aguda de crónica debido a la naturaleza distinta de ambas, sin embargo, existen varias publicaciones en las que se utiliza el diagnóstico de "rinosinusitis" sin diferenciarlas.

\section{Rinosinusitis aguda (RSA)}

De 1.589 pacientes sometidos a trasplante renal solo el $0,13 \%$ de ellos presentó RSA previo al procedimiento y un $0,2 \%$ postrasplante, sin recurrencia de la enfermedad ${ }^{1}$, este porcentaje es inferior a los datos de la guía EPOS 2012, con una prevalencia del 6\%-12\%, y recurrencia del $0,035 \% 5$. En el caso de RSA, la evaluación sinonasal previa al trasplante no pudo predecir el curso clínico posterior al trasplante. En un estudio de 4.562 pacientes sometidos a trasplante de órgano sólido (riñón, hígado, pulmón y corazón) menos del 1\% de ellos desarrolló una RSA que requiriera hospitalización y ninguno de ellos reportó complicaciones, siendo $H$. influenzae la bacteria más frecuentemente involucrada ${ }^{6}$. Sólo 2 pacientes del total estudiado presentaron RSA fúngica invasiva (RSFI). Sin embargo, Dhong y cols. ${ }^{7}$ reportan una incidencia del $10 \%$ de RSFI en una serie de pacientes que incluía aquellos con trasplante de médula ósea, quienes son mucho más susceptibles al desarrollo de enfermedades fúngicas producto de los efectos secundarios a la neutropenia, como reducida actividad fagocítica y pobre recuperación de células Natural Killer, entre otros ${ }^{8}$.

\section{Recomendaciones en RSA}

Los pacientes con RSA necesitan un tratamiento médico adecuado, independientemente del estado del trasplante, siendo necesario que la RSA esté en remisión completa justo antes del trasplante. El diagnóstico se basa en los criterios de la guía EPOS 2012, siendo in- necesario el uso de imágenes complementarias en pacientes asintomáticos.

\section{Rinosinusitis crónica (RSC)}

En el estudio de Ryu G. y cols. ${ }^{1}, 1.589$ pacientes recibieron evaluación rinosinusal pretrasplante renal, la cual consistía en una detallada historia clínica junto con endoscopía nasal y en caso de encontrar hallazgos anormales en alguno de ellos o ambos, evaluación con tomografía computarizada de cavidades paranasales (TC de CPN). Los investigadores llegaron a la conclusión de que la prevalencia de sinusitis aguda y sinusitis crónica con y sin pólipos no aumenta luego del trasplante, siendo necesario el tratamiento de aquellos pacientes sintomáticos, sin beneficio de tratamiento en aquellos pacientes asintomáticos con hallazgos patológicos en la TC. En un estudio retrospectivo de 996 pacientes que recibieron trasplante hepático, 28 de ellos presentaron rinosinusitis pretrasplante (26 RSC y 2 sospechas de RSA fúngica invasiva), correspondiendo a una prevalencia del $2,6 \%$. $22 / 28$ pacientes no recibieron ningún tipo de tratamiento y estos pacientes presentaron un agravamiento de los síntomas postrasplante sin contribuir al aumento de la mortalidad infecciosa y global'.

En pacientes receptores de células hematopoyéticas la presencia de síntomas de rinosinusitis se asocia a la recurrencia de síntomas rinosinusales posterior al trasplante $(\mathrm{p}=0,015)$, con una tendencia a la disminución en caso de recibir un tratamiento óptimo ${ }^{10}$. En otro estudio con 71 receptores de células hematopoyéticas, 4 presentaron rinosinusitis crónica pretrasplante, los cuales recibieron tratamiento médico asociado en 3 casos a cirugía endoscópica funcional (CEF). Ninguno de ellos requirió postrasplante. Por otro lado, de todos los pacientes incluidos en el estudio, sólo 2 presentaron RS postrasplante, un caso de RSA y un caso de RS odontogénica, y ninguno de ellos poseía síntomas previos ni endoscopía nasal sugerente de rinosinusitis en la evaluación inicial ${ }^{11}$. En ambos estudios la aparición de hallazgos imagenológicos en pacientes asintomáticos no se asocia al desarrollo de rinosinusitis postrasplante. Tzelnick y cols. ${ }^{6}$ reportan una prevalencia de RSC del $0,7 \%$ en pacientes sometidos a trasplante de órgano sólido. En 
cuanto a la microbiología, las pseudomonas fueron los patógenos más frecuentemente encontrados con una frecuencia de hasta el $40 \%$. Ninguno de estos pacientes presentó síntomas previos al trasplante. Como es de notar en los estudios mencionados previamente, aquellos pacientes con hallazgos anormales imagenológicos, pero sin síntomas de rinosinusitis, no muestran un deterioro clínico posterior al trasplante. Concordante con esto un estudio demostró que del total de TC de CPN patológicos en estudio pretrasplante, un $22,8 \%$ de los pacientes presentaba rinosinusitis sintomática, mientras que el $77,2 \%$ restante no presentaba ninguna sintomatología, no recomendando la evaluación rutinaria imagenológica debido a la alta tasa de falsos positivos ${ }^{12}$.

\section{Flujograma y recomendaciones en evalua- ción rinosinusal}

Recomendamos que previo a todo trasplante debe realizarse una evaluación rinosinusal, la cual consiste en una exhaustiva historia clínica y endoscopía nasal. En el caso de que identificar

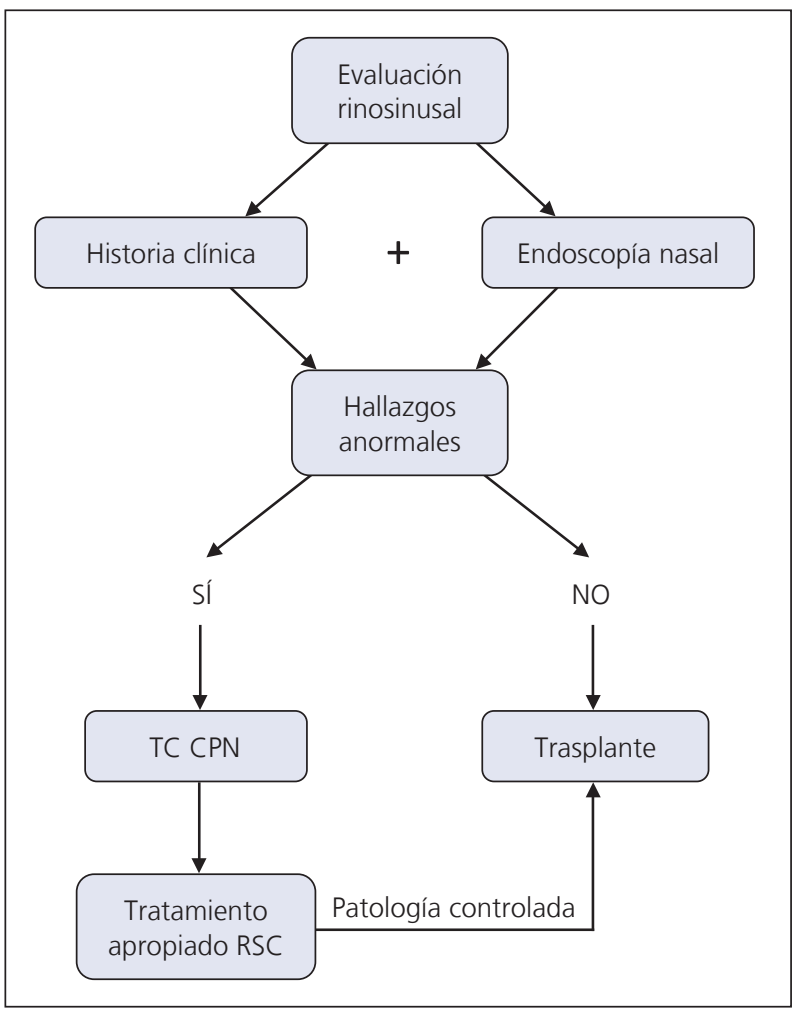

Figura 1. Flujograma de evaluación rinosinusal pretrasplante. anomalías en alguna o ambas, debe realizarse una TC de CPN para certificar el diagnóstico y otorgar un tratamiento apropiado previo al trasplante. Por el contrario, en caso de que la historia clínica y la endoscopía nasal sean normales, el paciente puede someterse al procedimiento, sin necesidad de estudios imagenológicos. En la Figura 1 se observa el flujograma propuesto. Debemos recalcar que en pacientes receptores de células hematopoyéticas la incidencia de RSFI es mayor al resto (hasta $63 \%{ }^{13}$ ), por lo tanto, es importante la sospecha diagnóstica en aquellos pacientes con inicio agudo de síntomas rinosinusales.

\section{Consideraciones en pacientes con trasplante pulmonar}

La rinosinusitis crónica es frecuente en pacientes con indicación de trasplante pulmonar, especialmente en aquellos pacientes con fibrosis quística (FQ), siendo más de la mitad de ellos sometidos a CEF previo al trasplan$t^{14}$. Esta asociación se ha explicado en base al concepto de vía aérea unificada y a que la descarga posterior puede inducir a la infección respiratoria baja, convirtiéndose en una fuente de infección durante la inmunosupresión postrasplante ${ }^{15}$. Distintos estudios demuestran que el tratamiento de la rinosinusitis tiene un efecto positivo en la prevención de infecciones postrasplante ${ }^{16-18}$, además se ha reportado que el drenaje crónico con patógenos en aloinjertos pulmonares es causado por el reservorio bacteriano de los senos paranasales ${ }^{19}$, siendo incluso genotípicamente idénticos ${ }^{20}$. En un estudio con 154 pacientes con FQ el 60\% presentaba el mismo patógeno aislado en el lavado nasal y bronquioalveolar. ${ }^{14}$ Los patógenos más frecuentemente involucrados son Ps. aeruginosa (prevalencia cercana al 84\%), Burkholderia cepacia y Staphylococcus aureus, demostrándose una alta correlación entre el cultivo sinusal pretrasplante tomado mediante endoscopía nasal y el cultivo obtenido de lavado bronquioalveolar postrasplante, asociándose además a una mala respuesta postrasplante $e^{21}$, bronquiolitis obliterante ${ }^{22}$, poliposis nasal ${ }^{23,24}$, y menor sobrevida ${ }^{25}$.

En 148 pacientes que recibieron trasplante pulmonar debido a diversas indicaciones, aquellos receptores que presentaban RSC previa al trasplante y que no recibieron cirugía endoscó- 
pica funcional el porcentaje de sobrevida global fue significativamente menor que aquellos que sí fueron sometidos a CEF, en estos últimos la curva de sobrevida se compara a la de los pacientes sin RSC con un seguimiento mayor a 12 años. En este mismo estudio no existieron diferencias significativas en las curvas de sobrevida al analizar aquellos receptores con y sin anormalidades imagenológicas en la $\mathrm{TC}^{15}$. De todos los pacientes sometidos a trasplante, la CEF es más frecuente en pacientes trasplantados pulmonares, con un mayor número de intervenciones por pacientes (más de 2$)^{6}$.

Se ha reportado un amplio rango en la presencia de síntomas de RS que va del 20,6\% al $62 \%{ }^{14}$, alteración de la nasofibroscopía en el $73,5 \%$ y un $93,5 \%$ en la TC CPN. La discordancia entre endoscopía/TC y clínica puede deberse a la adaptación a los síntomas y desvaloración en comparación a otras manifestaciones más severas de la enfermedad ${ }^{26}$, por lo tanto, se recomienda realizar una TC en la evaluación de pacientes con FQ, aunque no existen estudios que demuestren que la conducta por imágenes en estos pacientes beneficie el resultado postrasplante y disminuya la mortalidad, siendo superior la endoscopía nasal para su estudio ${ }^{18}$. Se ha observado, además, que la CEF en pacientes trasplantados de pulmón, aumenta significativamente el volumen espiratorio forzado del primer segundo (VEF1) y disminuye el promedio de días de hospitalización debido a bronconeumonía de 32,82 a 5,41 días, en una serie de 16 pacientes en comparación a los 6 meses previos a la $\mathrm{CEF}^{27}$. Además, se ha visto que la CEF disminuye la frecuencia del uso de antibióticos por infecciones respiratorias en estos pacientes ${ }^{28}$.

\section{Recomendaciones en pacientes con trasplante pulmonar}

La literatura avala una conducta agresiva en el tratamiento de rinosinusitis, tanto médico como quirúrgico, ya que la colonización de las cavidades paranasales induce el deterioro de la función pulmonar ${ }^{27}$. Tomando en cuenta que estos pacientes habitualmente cursan repetidas infecciones, con tratamiento antibióticos prolongados y exposición a patógenos hospitalarios, se sugiere tomar cultivos para ajustar la terapia antibiótica y mejorar la respuesta al trasplante pulmonar. Considerando el estudio previamente realizado en que se demuestra una alta correlación entre los patógenos encontrados en el lavado broncoalveolar postrasplante y el cultivo sinusal pretrasplante ${ }^{21}$, sugerimos realizar toma de cultivo de meato medio de forma endoscópica in office previo al trasplante pulmonar. En caso de que el procedimiento no sea adecuadamente tolerado por el paciente, recomendamos hacerlo en pabellón con anestesia general utilizando el mismo acto quirúrgico en que se realizará el trasplante.

\section{Evaluación otológica}

\section{Otitis media aguda}

No existe literatura específica sobre otitis media aguda (OMA) en pacientes trasplantados, sin embargo, las infecciones agudas deberían ser tratadas previamente a intervenciones quirúrgicas, lo cual debe ser reforzado aún más en aquellos pacientes que irán a trasplante e inmunosupresión. Extrapolando datos de pacientes inmunosuprimidos por otras razones, los pacientes trasplantados deberían recibir un adecuado diagnóstico y tratamiento agresivo en casos de OMA, con el fin de prevenir complicaciones potencialmente graves ${ }^{27}$.

\section{Otitis media crónica simple y colesteatomatosa}

En comparación a la RSC, la presencia de otitis media crónica (OMC) es aún menos frecuente en pacientes trasplantados. En la literatura solo existe un estudio disponible realizado en 3.278 pacientes receptores de órganos sólidos, de los cuales 65 (2\%) presentaban perforación timpánica. Los pacientes de acuerdo a la clínica fueron separados en tres grupos: 25 de los pacientes tenían OMC simple no supurada, estos fueron seguidos en el tiempo sin recibir tratamiento previo al trasplante; 26 presentaban OMC simple supurada, los cuales recibieron solo tratamiento antibiótico previo al trasplante. Del resto 14 fueron sometidos a cirugía, ya que presentaban historia de otorrea más TC alterada (probablemente debido a colesteatoma). En aquel grupo que fue sometido a intervención quirúrgica la presencia de otorrea postrasplante fue significativamente menor que el grupo tratado con antibióticos por OMC supurada y aquel bajo observación por OMC no supurada ${ }^{28}$. 


\section{Flujograma y recomendaciones en evaluación otológica}

Actualmente no existe literatura disponible para generar recomendaciones en base a la evidencia, por lo tanto, el criterio clínico debe primar. En la Figura 2 se observa flujograma propuesto. Nuestras recomendaciones son:

OMA: Tratar previo a trasplante.

OMC simple no supurada y OMC fibroadhesiva: No posponer trasplante, manejo habitual.

OMC simple supurada: Tratar sobreinfección de acuerdo a manejo local. Toma de cultivos en caso de no haber respuesta a tratamiento.

OMC colesteatomatosa: Con otorrea y/o complicada intervención quirúrgica previa al trasplante. Sin otorrea puede diferirse tratamiento de acuerdo a decisión clínica.

\section{Medicamentos ototóxicos}

En el manejo de los pacientes trasplantados es necesario el uso de distintos tipos de fármacos. Ciclosporina, tacrolimus, micofenolato y prednisona son inmunosupresores habituales en estos pacientes, todos ellos con efectos ototóxicos reportados ${ }^{29}$. Además, es importante considerar que estos pacientes están expuestos a un uso mayor de antibióticos tanto pre como postrasplante. En un estudio realizado en 27 pacientes pediátricos con trasplante renal, 17 de ellos presentaban hipoacusia sensorioneural mayor en tonos agudos y 8 presentaron disminución de la comprensión del habla, encontrándose que el nivel de ciclosporina fue significativamente mayor en pacientes con hipoacusia y disminución de la comprensión del habla ${ }^{30}$. Hasta el momento no existen guías de seguimiento para estos pacientes, creemos que la pauta de seguimiento audiométrico debe ser individualizada caso a caso de acuerdo a los fármacos utilizados y antecedentes del paciente, idealmente con audiometría previo al inicio del uso de estos fármacos.
Figura 2. Flujograma de evaluación otológica pretrasplante.

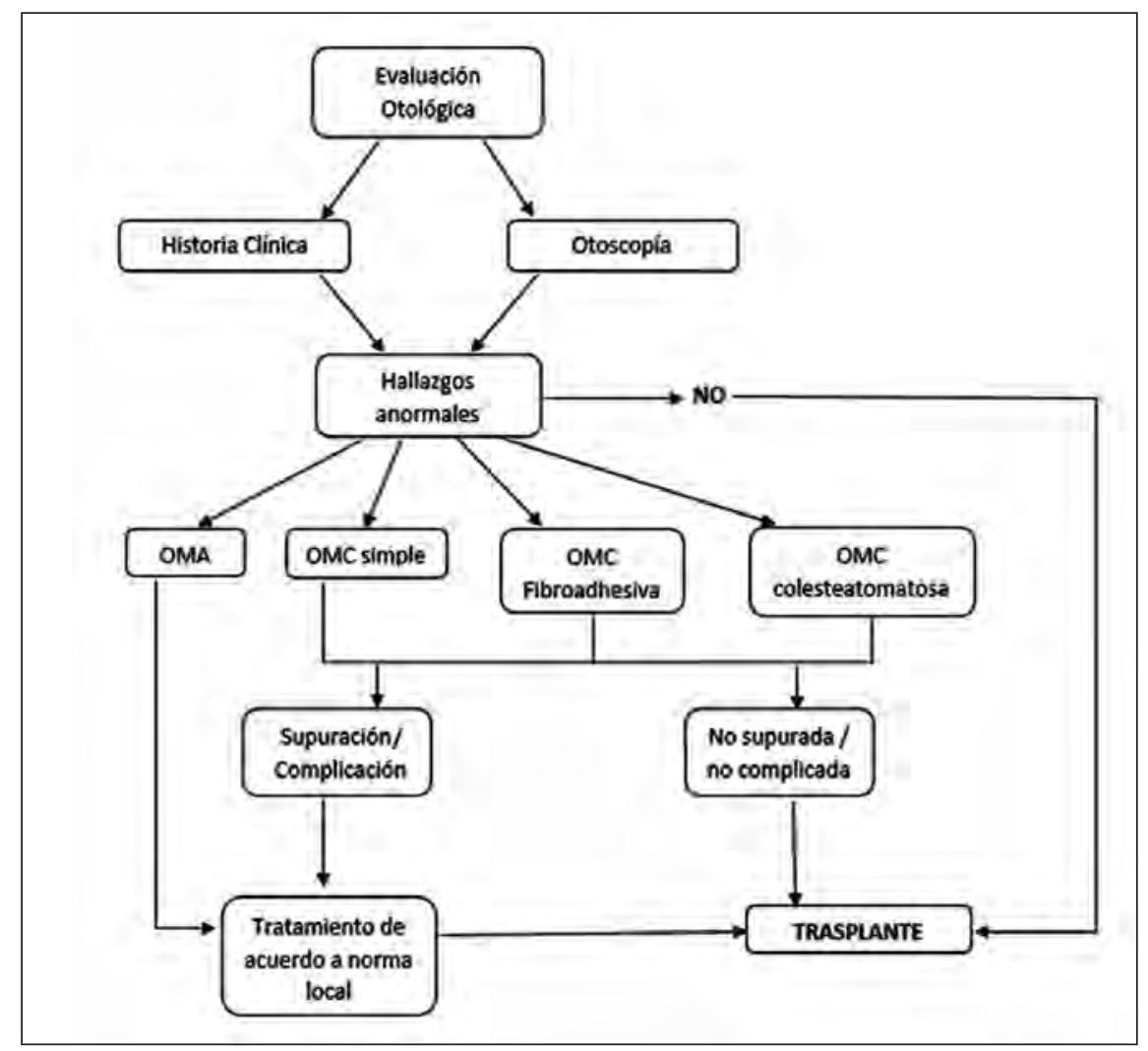




\section{Evaluación faringoamigdalina}

La literatura acerca del impacto de la patología faringoamigdalina en pacientes trasplantados es prácticamente nula, al igual que lo previamente expuesto creemos que las infecciones agudas deberían ser tratadas previamente a cualquier tipo de trasplante. El aumento de la sobrevida de pacientes pediátricos sometidos a trasplante de órganos sólidos también ha aumentado las complicaciones de la inmunosupresión como la enfermedad linfoproliferativa postrasplante (ELP), siendo esta la malignidad secundaria más frecuente en niños con una mortalidad del $10 \%-20 \%{ }^{31-34}$. La incidencia de ELP en niños es mayor al 15\%, presentándose en el 63\% de los casos en cabeza y cuello, especialmente en el anillo de Waldeyer y cuya forma de manifestación es la hiperplasia adenoamigdalina ${ }^{35}$. En adultos su incidencia es menor, con un rango de $1 \%$ al $10 \%{ }^{36}$.

Se requiere un diagnóstico oportuno de ELP, ya que en etapas tempranas la disminución de la inmunosupresión puede revertir la enfermedad. Sin embargo, el diagnóstico es un desafío considerando la alta prevalencia de hiperplasia adenoamigdalina en niños. El cuadro clínico es variable y puede presentar síntomas poco específicos como fiebre, compromiso del estado general y baja de peso, además de presentar síntomas relacionados al sitio donde se produce ELP. La presencia de estos síntomas sistémicos en el contexto de una hiperplasia adenoamigdalina, pueden corresponder a banderas rojas de ELP, así como también un crecimiento acelerado tonsilar asociado a la presencia de adenopatías. De todas formas, muchas veces el cuadro puede ser asintomático, sobre todo en estadios iniciales ${ }^{37}$. El diagnóstico definitivo de ELP es histopatológico mediante adenoamigdalectomía. En pacientes sin sospecha de ELP la incidencia de esta patología en tejidos fue de un $5,7 \%{ }^{35}$.

\section{Discusión}

Hasta el momento existe escasa literatura acerca de la evaluación otorrinolaringológica pretrasplante, así como también de las complicaciones infecciosas, debido probablemente a que la patología infecciosa de cabeza y cuello no es la preponderante en estos pacientes. Sin embargo, en caso de patología aguda, estos datos posiblemente estén subestimados debido a que muchas veces los pacientes no consultan en centros terciarios de donde se obtienen las bases de datos. En el área rinosinusal la anamnesis junto con un adecuado examen físico que incluya endoscopía nasal es el método apropiado, existe consenso en que el estudio radiológico no debe solicitarse en la evaluación inicial, destinándose solo a pacientes en que se sospeche alguna patología rinosinusal por historia clínica y/o examen físico.

En cuanto a OMC, no existe evidencia contundente para establecer directrices. De acuerdo a las publicaciones y nuestra opinión, la conducta apropiada sería un tratamiento adecuado y oportuno según las normas locales, sin necesidad de diferir el trasplante a menos que existan signos de complicación. Es importante mencionar que muchos fármacos utilizados en estos pacientes tales como ciclosporina, tacrolimus, micofenolato, entre otros, son ototóxicos reportados, por lo tanto, se hace necesario un seguimiento audiométrico individualizado e, idealmente, un estudio previo al inicio de estos fármacos, especialmente en aquellos pacientes con uso de ciclosporina.

La literatura en patología faringoamigdalina en pacientes trasplantados es prácticamente nula. Sin embargo, siguiendo la lógica de la conducta en infecciones agudas, estas deberían ser tratadas previamente a cualquier tipo de trasplante. El aumento de la sobrevida de pacientes pediátricos sometidos a trasplante de órganos sólidos también ha aumentado las complicaciones de la inmunosupresión como la enfermedad linfoproliferativa postrasplante (ELP), siendo la malignidad secundaria más frecuente en niños. El desafío es llegar a un diagnóstico oportuno de ELP, ya que en etapas tempranas la disminución de la inmunosupresión puede revertir la enfermedad.

En general, para las distintas patologías el diagnóstico y tratamiento es el estándar de acuerdo a las guías clínicas y normas locales, no obstante, específicamente en el grupo de pacientes con trasplante de pulmón el enfrentamiento farmacológico y quirúrgico es más agresivo, requiriendo habitualmente varias intervenciones, ya que los senos paranasales actúan como reservorio permitiendo el descenso y recolonización del pulmón trasplantado. 


\section{Conclusión}

Una adecuada anamnesis y examen físico son los pilares de la evaluación pretrasplante en otorrinolaringología, reservándose el estudio con imágenes para aquellos pacientes con alteraciones sospechosas. Si bien, el manejo de las patologías corresponde al mismo que se realiza habitualmente en otros pacientes, el criterio médico es relevante para individualizar las pautas de tratamiento y seguimiento de acuerdo a la patología y características propias del paciente, siendo necesaria una conducta más agresiva en aquellos sometidos a trasplante pulmonar.

\section{Bibliografía}

1. Ryu G, Seo MY, Lee KE, et al. Clinical course of rhinosinusitis and efficacy of sinonasal evaluation in kidney transplant recipients: review of 1589 patients. Eur Arch Otorhinolaryngol 2018;275:1183-1188. Disponible en https://doi.org/10.1007/s00405-0184941-4.

2. Kim YJ, Yoon JH, Kim SI, et al. Impact of Pretransplant Infections on Clinical Course in Liver Transplant Recipients. Transplant Proc 2018;50:11531156. Disponible en https://doi.org/10.1016/j. transproceed.2018.01.036.

3. Sun HY, Cacciarelli TV, Singh N. Impact of pretransplant infections on clinical outcomes of liver transplant recipients. Liver Transplant 2010;16:222228. Disponible en https://doi.org/10.1002/lt.21982.

4. Eguchi S, Soyama A, Takatsuki M, et al. The Impact of Treated Bacterial Infections within One Month before Living Donor Liver Transplantation in Adults. Ann Transplant 2014;19:674-679. Disponible en https://doi.org/10.12659/AOT.892095.

5. Fokkens WJ, Lund VJ, Mullol J, et al. EPOS 2012: European position paper on rhinosinusitis and nasal polyps 2012. A summary for otorhinolaryngologists. Rhinol J 2012;50:1-12. Disponible en https://doi. org/10.4193/Rhino50E2.

6. Tzelnick S, Soudry E. Rhinosinusitis in Solid Organ Transplant Recipients: Analysis of 4562 Transplanted Patients. Am J Rhinol Allergy 2019;33:56-61. Disponible en https://doi. org/10.1177/1945892418808717.

7. Dhong HJ, Lee JC, Ryu JS, Cho DY. Rhinosinusitis in transplant patients. Clin Otolaryngol Allied Sci 2001;26:329-333. http://www.ncbi.nlm.nih.gov/ pubmed/11559348.

8. Stuehler C, Kuenzli E, Jaeger VK, et al. Immune Reconstitution After Allogeneic Stem Cell
Transplantation and Association With Occurrence and Outcome of Invasive Aspergillosis. J Infect Dis 2015;212:959-967. Disponible en https://doi. org/10.1093/infdis/jiv143.

9. Moon BJ, Han JH, Jang YJ, Lee B-J, Chung Y-S. Effect of chronic rhinosinusitis on liver transplant patients. Am J Rhinol Allergy 2009;23:492-496. Disponible en https://doi.org/10.2500/ajra.2009.23.3352.

10. Won Y-W, Yi SY, Jang JH, et al. Retrospective analysis of paranasal sinusitis in patients receiving hematopoietic stem cell transplantation. Int $J$ Hematol 2011;93:383-388. Disponible en https:/doi. org/10.1007/s12185-011-0797-8.

11. Moeller CW, Martin J, Welch KC. Sinonasal Evaluation Preceding Hematopoietic Transplantation. Otolaryngol Neck Surg 2011;144:796-801. Disponible en https://doi.org/10.1177/0194599810395089.

12. Tomazic P, Neuschitzer A, Koele W, Lang-Loidolt D. Feasibility of routine paranasal sinus CT-scans in preoperative transplant patients. Ann Transplant 2011;16:31-35.

13. Ortiz E, Ng RTY, Alliegro FC, Teixeira C, Muranaka EB, Sakano E. Microbiology of rhinosinusitis in immunosupressed patients from the University Hospital. Braz J Otorhinolaryngol 2011;77:522-525.

14. Morlacchi LC, Greer M, Tudorache I, et al. The burden of sinus disease in cystic fibrosis lung transplant recipients. Transpl Infect Dis 2018;20:e12924. Disponible en https://doi. org/10.1111/tid.12924.

15. Kariya S, Okano M, Oto T, et al. The impact of chronic rhinosinusitis on long-term survival in lung transplantation recipients. Acta Otolaryngol 2017;137:529-533. Disponible en https://doi.org/10.1 080/00016489.2016.1247497.

16. Vital D, Hofer M, Benden C, Holzmann D, Boehler A. Impact of sinus surgery on pseudomonal airway colonization, bronchiolitis obliterans syndrome and survival in cystic fibrosis lung transplant recipients. Respiration 2013;86:25-31. Disponible en https://doi. org/10.1159/000339627.

17. Holzmann D, Speich R, Kaufmann T, et al. Effects of sinus surgery in patients with cystic fibrosis after lung transplantation: a 10-year experience. Transplantation 2004; 77:134-136. Disponible en https://doi. org/10.1097/01.TP.0000100467.74330.49.

18. Gutiérrez C, Ribalta G, Largo I. Análisis retrospectivo de pacientes portadores de rinosinusitis crónica por fibrosis quística. Acta Otorrinolaringol Esp 2012;63:286-291. Disponible en https://doi. org/10.1016/j.otorri.2012.04.005.

19. Walter S, Gudowius P, Bosshammer J, et al. Epidemiology of chronic Pseudomonas aeruginosa infections in the airways of lung transplant recipients with cystic fibrosis. Thorax 1997; 52:318-321. Disponible en https://doi.org/10.1136/ thx.52.4.318. 
20. Mainz JG, Hentschel J, Schien C, et al. Sinonasal persistence of Pseudomonas aeruginosa after lung transplantation. J Cyst Fibros 2012; 11:158161. Disponible en https://doi.org/10.1016/j. jcf.2011.10.009.

21. Choi KJ, Cheng TZ, Honeybrook AL, et al. Correlation between sinus and lung cultures in lung transplant patients with cystic fibrosis. Int Forum Allergy Rhinol 2018; 8:389-393. Disponible en https:// doi.org/10.1002/alr.22067.

22. Botha P, Archer L, Anderson RL, et al. Pseudomonas aeruginosa colonization of the allograft after lung transplantation and the risk of bronchiolitis obliterans syndrome. Transplantation 2008; 85:771-774. Disponible en https://doi.org/10.1097/ TP.0b013e31816651de.

23. Vital D, Holzmann D, Boehler A, Hofer M. Nasal polyposis in lung transplant recipients with cystic fibrosis. J Cyst Fibros 2013; 12:266-270. Disponible en https://doi.org/10.1016/j.jcf.2012.08.013.

24. Boari L, de Castro Júnior NP. Diagnosis of chronic rhinosinusitis in patients with cystic fibrosis: correlation between anamnesis, nasal endoscopy and computed tomography. Braz J Otorhinolaryngol 2005; 71:705-710.

25. Ramos BF, Pinna F de R, Campos SV, et al. Assessment of Pulmonary Function before and after Sinus Surgery in Lung Transplant Recipients. Int Arch Otorhinolaryngol 2018; 22:157-160. Disponible en https://doi.org/10.1055/s-0037-1606600.

26. Cheng TZ, Choi KJ, Honeybrook AL, et al. Decreased Antibiotic Utilization After Sinus Surgery in Cystic Fibrosis Patients With Lung Transplantation. Am J Rhinol Allergy 2019; 33:354-358. Disponible en https://doi.org/10.1177/1945892419830624.

27. Hildrew D, Adams A, Winters R, Aslam R. Management of Complications of Acute Otomastoiditis in Solid Organ Transplant Patients. J La State Med Soc 2016; 168:104-106.

28. Yu MS, Kim HC, Ahn JH. Management and Outcomes of Chronic Otitis Media in Patients Who Received Solid Organ Transplantation. Otolaryngol Neck Surg 2012; 146:567-571. Disponible en https:// doi.org/10.1177/0194599811433276.
29. Broto PL, Brumós LG. ACÚFENOS Y FÁRMACOS; 2018. https://www.acúfenos.org/docs/Acúfenos_y_ Farmacos.pdf. Accessed August 23, 2019.

30. Gulleroglu K, Baskin E, Aydin E, Ozluoglu L, Moray G, Haberal M. Hearing status in pediatric renal transplant recipients. Exp Clin Transplant 2015;13(4):324-328. Disponible en https://doi. org/10.6002/ect.2014.0158.

31. LaRosa C, Jorge Baluarte H, Meyers KEC. Outcomes in pediatric solid-organ transplantation. Pediatr Transplant 2011;15:128-141. Disponible en https:// doi.org/10.1111/j.1399-3046.2010.01434.x

32. Parker A, Bowles K, Bradley JA, et al. Management of post-transplant lymphoproliferative disorder in adult solid organ transplant recipients - BCSH and BTS Guidelines. Br J Haematol 2010;149:693-705. Disponible en https://doi.org/10.1111/j.13652141.2010.08160.x

33. Roy S, Vivero RJ, Smith LP. Adenotonsillar pathology in post-transplant patients. Int J Pediatr Otorhinolaryngol 2008;72:865-868. Disponible en https://doi.org/10.1016/J.IJPORL.2008.02.016.

34. Dharnidharka VR, Martz KL, Stablein DM, Benfield MR. Improved Survival with Recent Post-Transplant Lymphoproliferative Disorder (PTLD) in Children with Kidney Transplants. Am J Transplant 2011;11:751-758. Disponible en https:// doi.org/10.1111/j.1600-6143.2011.03470.x.

35. Roberts J, Powell J, Mather MW, Powell S, Brodlie $\mathrm{M}$. A review of adenotonsillar hypertrophy and adenotonsillectomy in children after solid organ transplantation. Int J Pediatr Otorhinolaryngol 2018;114:29-35. Disponible en https://doi. org/10.1016/j.ijporl.2018.08.020.

36. Shapiro R, Nalesnik M, McCauley J, et al. Posttransplant lymphoproliferative disorders in adult and pediatric renal transplant patients receiving tacrolimus-based immunosuppression. Transplantation 1999;68:1851-1854. Disponible en https://doi.org/10.1097/00007890-199912270-00006

37. Hussein K, Tiede C, Maecker-Kolhoff B, Kreipe H. Posttransplant Lymphoproliferative Disorder in Pediatric Patients. Pathobiology 2013;80:289-296. Disponible en https://doi.org/10.1159/000350331. 